\title{
Processus de contextualisation et développement de la compétence scripturale de justification d'élèves du premier cycle du secondaire
}

\section{First-cycle secondary students' contextualization process and the development of the scriptural competency of justification Proceso de contextualización y desarrollo de la competencia escrita de justificación en alumnos de primer ciclo de secundaria}

\section{Marie-Hélène Forget}

Volume 16, numéro 2, 2013

URI : https://id.erudit.org/iderudit/1029141ar

DOI : https://doi.org/10.7202/1029141ar

\section{Aller au sommaire du numéro}

Éditeur(s)

Faculté d'éducation, Université de Sherbrooke

ISSN

1911-8805 (numérique)

Découvrir la revue

Citer cet article

Forget, M.-H. (2013). Processus de contextualisation et développement de la compétence scripturale de justification d'élèves du premier cycle du secondaire. Nouveaux cahiers de la recherche en éducation, 16(2), 29-55. https://doi.org/10.7202/1029141ar
Résumé de l'article

Une recherche doctorale qualitative et exploratoire portant sur l'apprentissage de l'écriture de justifications en classe de français chez des élèves plurilingues de 12 à 14 ans a révélé des liens entre la contextualisation qu'effectue un élève d'une situation d'écriture et sa manière d'élaborer le propos de sa justification. En effet, selon que l'élève tienne compte des paramètres de la situation fictive d'écriture ou qu'il focalise plutôt son attention sur les exigences scolaires, il parviendra plus ou moins bien à tenir compte de la dimension dialogique inhérente au discours justificatif. Cet article illustre ces liens à partir d'extraits d'entretiens et met en évidence des besoins d'apprentissage liés à ce processus de contextualisation qui, s’il est bien mené, soutient l'élève dans le développement de sa compétence scripturale de justification. 


\section{Processus de contextualisation et développement de la compétence scripturale de justification d'élèves du premier cycle du secondaire}

\section{Marie-Hélène Forget}

Université du Québec à Montréal

\section{Résumé}

Une recherche doctorale qualitative et exploratoire portant sur l'apprentissage de l'écriture de justifications en classe de français chez des élèves plurilingues de 12 à 14 ans a révélé des liens entre la contextualisation qu'effectue un élève d'une situation d'écriture et sa manière d'élaborer le propos de sajustification. En effet, selon que l'élève tienne compte des paramètres de la situation fictive d'écriture ou qu'il focalise plutôt son attention sur les exigences scolaires, il parviendra plus ou moins bien à tenir compte de la dimension dialogique inhérente au discours justificatif. Cet article illustre ces liens à partir d'extraits d'entretiens et met en évidence des besoins d'apprentissage liés à ce processus de contextualisation qui, s'il est bien mené, soutient l'élève dans le développement de sa compétence scripturale de justification.

Mots-clés: didactique, contextualisation, apprentissage, écriture, justification

\section{First-cycle secondary students' contextualization process and the development of the scriptural competency of justification}

\section{Abstract}

A qualitative and exploratory doctoral study on learning to write justifications in French class among 12-14 year old plurilingual students has revealed links between students' contextualization of a writing situation, on one hand, and the way they 
develop their justifications, on the other. Indeed, depending on whether the student takes into account parameters of the fictional writing situation or instead focuses on the academic requirements, he or she will, to differing degrees, be able to successfully take into account the dialogical dimension inherent to the discourse of justification. The present article illustrates these links based on interview excerpts and highlights the need for learning associated with this contextualization process, which, if properly carried out, supports students in developing the scriptural competency of justification.

Keywords: didactics, contextualization, learning, writing, justification

\section{Proceso de contextualización y desarrollo de la competencia escrita de justificación en alumnos de primer ciclo de} secundaria

\section{Resumen}

Una investigación doctoral cualitativa y exploratoria referente al aprendizaje de la escritura de justificación en clase de francés con alumnos plurilingües de 12 a 14 años reveló que existen vínculos entre la contextualización realizada por un alumno en situación de escritura y su manera de elaborar el contenido de su justificación. En efecto, según el alumno tiene en cuenta parámetros de la situación ficticia de escritura en vez de centrar su atención en las exigencias escolares, logrará más o menos bien tener en cuenta la dimensión dialógica inherente al discurso justificativo. Este artículo ilustra estos vínculos a partir de extractos de entrevistas y destaca necesidades de aprendizaje unidos a este proceso de contextualización, el cual, de ser bien conducido, apoya al alumno en el desarrollo de su competencia escrita de justificación.

Palabras clave: didáctica, contextualización, aprendizaje, escritura, justificación

\section{Introduction}

Dans la foulée de la réforme québécoise de l'éducation amorcée à l'ordre primaire, le ministère de l'Éducation, du Loisir et du Sport (MÉLS) prescrit un nouveau programme de 
français langue d'enseignement pour le $1^{\text {er }}$ cycle du secondaire ${ }^{1}$ (MÉLS, 2004). Des savoirs à enseigner sont alors ajoutés, dont le genre discursif justificatif, considéré dans ce programme comme une "sensibilisation à l'argumentation" (p. 110). Cinq ans plus tard, soit en juin 2009, le Ministère introduit une nouvelle épreuve nationale d'écriture à la fin du $1^{\text {er }}$ cycle secondaire. On soumet aux élèves une tâche d'écriture dans laquelle ils doivent entre autres "appuyer [leurs] propos en élaborant des justifications" (MÉLS, 2009). Avec deux épreuves d'écriture de fin de cycle au secondaire, l'une évaluant au $1^{\text {er }}$ cycle les compétences justificatives des élèves, l'autre évaluant au $2^{\mathrm{e}}$ cycle leurs compétences argumentatives, il apparait que la maitrise de l'écrit à caractère argumentatif est une priorité pour les instances ministérielles qui en prescrivent, depuis lors, l'enseignement dès l'entrée au secondaire.

Ces nouvelles orientations tranchaient avec les pratiques habituelles des enseignants qui, en écho au programme précédent (MÉQ, 1995), concentraient surtout le travail sur les discours à caractère argumentatif dans les dernières années du secondaire. Aussi, des questions se sont posées dans les milieux de pratique² à propos de la nature de ce nouveau savoir (Qu'est-ce que justifier?), des pratiques enseignantes à instaurer en classe de français (Comment enseigner la justification?) ainsi que des capacités des élèves du $1^{\text {er }}$ cycle du secondaire à débuter l'apprentissage de la justification à l'écrit (les élèves sont-ils prêts pour cet apprentissage?). Sur ce dernier point, plusieurs recherches montrent que les élèves qui entrent au secondaire possèdent déjà une certaine expérience de la justification (GarciaDebanc, 1996; Goetz et Shatz, 1999; Golder, 1996; Kyratzis, Shuqum-Ross, et KoymenBahar, 2010). Comme on admet aujourd'hui en didactique des langues que les savoirs d'expérience s'articulent inévitablement aux savoirs enseignés à l'école (Reuter, 2001), cette recherche doctorale ${ }^{3}$ a visé à mieux connaître ceux des élèves en matière de justification

1 Le secondaire québécois suit les six années de l'école primaire et accueille les élèves à compter de l'âge de 12 ans. Le $1^{\text {er }}$ cycle du secondaire correspond aux deux premières années du cours secondaire qui compte cing années au total.

2 À cette époque, j'œuvrais à titre de conseillère pédagogique de français au secondaire dans une commission scolaire de la région montréalaise. Plusieurs enseignantes et enseignants ont alors émis des réserves quant aux capacités des élèves de ce cycle à faire face à ces nouvelles exigences, alléguant que, même si leurs élèves pouvaient assez aisément justifier leurs propos à l'oral, ils n'avaient peut-être pas la maturité intellectuelle nécessaire pour mener une telle conduite à l'écrit. Un avis syndical à l'égard de cette nouvelle épreuve a également éte publié dans Le Devoir et La Presse.

3 Cette recherche doctorale, dirigée par le Pr Olivier Dezutter de l'Université de Sherbrooke (Québec, Canada) et codirigée par la Pre Cécile Sabatier de l'Université Simon Fraser (Colombie-Britannique, Canada), a été financée dans le cadre du programme de bourses doctorales de l'Action concertée MÉLS -FRQ-SC, recherche sur l'écriture (2009-2012). 
et à mieux comprendre comment, du point de vue des élèves eux-mêmes, ces "déjà-là" pouvaient entrer en jeu lors de l'apprentissage de l'écriture de justification en classe de français. Des réponses à ces questions de recherche allaient pouvoir permettre de proposer des pratiques enseignantes qui tiennent compte des "déjà-là" et des pratiques effectives des élèves.

L'analyse par théorisation ancrée (Paillé et Mucchielli, 2008) de données d'entretien a permis de dégager plusieurs conceptions et de nombreuses pratiques de justifications qui témoignent d'un certain savoir-faire chez les élèves ayant participé à l'étude. Plus précisément, l'analyse de l'explicitation d'une activité d'écriture de justification réalisée en classe de français a révélé comment ces élèves contextualisent les situations d'écriture de justifications qui leur sont soumises et comment cette contextualisation influence leurs manières d'élaborer leurs justifications. Le présent article vise à en rendre compte.

\section{Capacités des élèves à justifier leurs propos: éléments de problématique}

Si la justification est considérée par plusieurs comme l'opération de base de l'argumentation (Adam, 1996; Fasel-Lauzon, 2014; Garcia-Debanc, 1996; Golder, 1996; Gombert, 2003), le discours proprement justificatif n'est pas, comme c'est le cas de I'argumentation, associé à une controverse. La justification se définit comme une conduite discursive qui vise à faire valoir, aux yeux d'un interlocuteur, la recevabilité, l'acceptabilité ou la légitimité d'un acte (Forget, 2012; Garcia-Debanc, 1996). Dans les interactions sociales du quotidien, la justification porte généralement sur un geste, un comportement, une parole ou un désir que l'on souhaite légitimer. Elle concerne donc très souvent les intérêts personnels de celui ou celle qui justifie (Goetz et Shatz, 1999). En classe de français, la justification porte plus spécifiquement sur un propos: des réponses à des questions qu'il faut justifier, des positions (avis, opinions) et des propositions (conseils, recommandations) (Garcia-Debanc, 1996; Masseron, 2003). On peut alors considérer, à la suite de Chartrand (2013), que la justification en classe de français est une conduite métadiscursive puisque «justifier ses paroles, c'est commenter son propre discours ou, plus précisément, dire pourquoi on affirme telle ou telle 
chose" (n.p.). Dans La progression des apprentissages au secondaire (MÉLS, 2011), on précise que la justification sert à "expliciter à postériori la démarche cognitive (le raisonnement) qui a conduit à l'expression du propos» (p. 21). Ajoutons, comme le souligne Fasel-Lauzon (2014), que la justification a une valeur subjective: elle concerne ce que croit, pense ou suggère une personne à propos d'une chose.

On sait que les premières conduites de justification se manifestent aussi tôt que vers 18 mois, avant même que l'enfant ne possède des savoirs langagiers et linguistiques permettant la production d'énoncés élaborés. Selon les recherches recensées, de telles capacités se développent principalement dans le cadre d'interactions orales informelles dans la famille, la sphère sociale et à l'école dès le primaire (Goetz et Shatz, 1999; Kyratzis et al., 2010).

D'autres recherches soulignent par ailleurs des difficultés pour les élèves de la fin du primaire et du début du secondaire à écrire des justifications conformes aux attentes scolaires relatives à l'écrit, leurs justifications étant plutôt construites sur le modèle de l'oral. Par exemple, Garcia-Debanc (1994) observe que les réponses écrites qui demandent une justification s'apparentent souvent au récit du raisonnement suivi pour parvenir à la réponse, comme si les élèves avaient retranscrit à l'écrit ce qu'ils auraient dit oralement. Des travaux ayant porté sur l'argumentation ont pu montrer une mise en place plus lente et laborieuse de conduites de type argumentatif à l'écrit par rapport à leur développement à l'oral (Brassart, 1990; Dolz, 1995; Golder, 1996; Kuhn et Udell, 2003; Wasim, 2007). Pour Brassart (1990), ce décalage serait dû à un développement spécifiquement textuel qui demande l'acquisition d'«une représentation progressivement plus complète et plus claire des propriétés du texte argumentatif» (p. 38); Golder (1996) évoque pour sa part la panoplie de genres textuels argumentatifs dont la maitrise des règles particulières s'avère difficile pour les élèves peu accoutumés à de tels usages; Gombert (1998) met en évidence certains facteurs influençant les performances des élèves scripteurs lors de l'écriture de justifications: des finalités communicatives et des thèmes éloignés des préoccupations des élèves ou le mode de gestion des discours (construits à plusieurs à l'oral, mais de manière autonome à l'écrit). De telles conclusions portent à penser que les spécificités du mode écrit constituent 
les principaux facteurs de difficultés en matière de justification et d'argumentation écrite à l'école. Garcia-Debanc (1996) pense toutefois que

la distinction essentielle porte moins sur la distinction entre oral et écrit que sur celle entre une argumentation dialogale où chacun tient sa place dans l'élaboration collective d'une justification au cœur de l'interaction (justification polygérée) et une argumentation monogérée, chaque fois que, à l'oral ou à l'écrit, l'élève se trouve en situation de produire un exposé long et suivi justifiant une prise de position (p. 118).

Selon l'auteure, une grande importance devrait donc être attribuée au travail sur les caractéristiques inhérentes à cette dimension dialogique des conduites de justification, tout particulièrement lorsqu'il s'agit de situations orales ou écrites gérées de manière autonome. Golder (1996) souligne d'ailleurs que la capacité à envisager une autre position que la sienne ainsi qu'à articuler ses arguments à ceux de l'interlocuteur constitue l'une des conditions de réussite de la production de discours argumentatifs à l'école et que cette "coopération" reste difficile pour les élèves âgés de 9 à 13 ans. Une question se pose alors sur la manière dont les élèves composent avec cette dimension dialogique intrinsèque à la justification, et plus largement sur la prise en compte des paramètres des situations d'écriture qui sont proposées aux élèves en classe de français.

\section{Contextualisation des situations de communication: assises conceptuelles}

Les interlocuteurs engagés dans une situation de communication vont gérer ensemble l'élaboration de leur discours dans le cours même de leur échange. Lorsque la situation de communication place l'énonciateur en position d'élaborer seul un discours néanmoins destiné à un lecteur ou à un auditeur, il produit alors son discours de manière autonome (Garcia-Debanc, 1996). Dans l'un ou l'autre de ces types de situations, les acteurs procèdent de manière plus ou moins consciente et spontanée à une lecture de la situation dans laquelle ils sont placés: ils en interprètent les paramètres, s'en construisent un sens et agissent en fonction de cette interprétation. Cette lecture interprétative se nomme le processus de contextualisation (Blanchet, 2009; Porquier et Py, 2004). 
Deux niveaux de paramètres situationnels doivent être considérés par les interlocuteurs, les locuteurs ou les scripteurs (Porquier et Py, 2004). Au niveau micro de l'énonciation, les paramètres vont concerner les variétés linguistiques d'usage, les contenus référentiels, les genres discursifs ainsi que les caractéristiques du média requis par la situation (échange verbal, message texte, lettre formelle, etc.) (Hornberger et Skilton-Sylvester, 2003). L'énonciateur va également devoir tenir compte de ses intentions communicationnelles, des enjeux énonciatifs et pragmatiques, du rôle et du statut des acteurs concernés, de leurs intérêts, valeurs et caractéristiques (Porquier et Py, 2004). Ce micro contexte est lui-même situé dans un milieu socioculturel qui l'englobe. Ce macro contexte concerne les pratiques sociales et langagières d'usage, les représentations sociales et les conventions institutionnelles (Blanchet, 2007) que doivent connaitre et utiliser les acteurs pour participer de manière légitime aux interactions sociales de ce milieu (Chevallard, 2003). La contextualisation étant un processus d'interprétation, les contingences micro et macro d'une situation donnée seront pour une part interprétées différemment par chacun selon l'empreinte de leurs "déjà-là" alors que d'autres seront comprises de la même manière par tous les acteurs concernés (Moore, Sabatier, Jacquet et Masinda, 2008; Porquier et Py, 2004).

Sur le plan des déterminations personnelles, la contextualisation d'une situation sera influencée par les "déjà-là", c'est-à-dire l'ensemble des expériences passées, des ressources et des pratiques langagières de l'acteur ainsi que divers rapports à de telles situations, à soi-même et aux autres ainsi qu'à l'objet du discours (Bulea et Bronckart, 2005). Ceux-ci conduisent chaque acteur à attribuer une pertinence à tel aspect de la situation plutôt qu'à tel autre dans la construction du contexte. Sur le plan des traits partagés par les interlocuteurs, ils vont s'imposer de la même manière pour tous les acteurs qui, évoluant dans un même milieu institutionnel, ont acquis une compréhension très similaire de certains paramètres (Bulea et Jeanneret, 2007; Porquier et Py, 2004). La contextualisation effectuée par un acteur va alors guider l'adoption d'une certaine stratégie cognitive et langagière appelée "posture» (Penloup, Chabanois et Joannidès, 2011), la mobilisation de certaines ressources ainsi que le recours à certaines pratiques sociales et langagières plutôt qu'à d'autres.

Les situations scolaires de communication ont ceci de particulier: elles visent spécifiquement l'apprentissage. Leurs paramètres renvoient à des éléments de cultures 
éducatives, linguistiques et didactiques et à leurs pratiques très prégnantes que les élèves doivent connaitre et auxquelles ils doivent se conformer pour fonctionner dans cette institution (Castellotti et Moore, 2008; Chevallard, 2003). Lors de l'enseignement et de l'apprentissage de l'écriture de textes longs, des textes justificatifs par exemple, l'élève doit tenir compte de deux situations de communication: celle de la classe, immédiate et réelle, qui implique l'enseignant et ses élèves et qui concerne un ou plusieurs objets d'apprentissage, puis celle de la tâche d'écriture, très souvent fictive, qui engage des interlocuteurs fictifs et qui sert de prétexte pour enseigner et apprendre à communiquer dans ce type de situations. Or, cette situation d'écriture, bien que fictive, comprend elle aussi deux niveaux de contextes que les élèves doivent contextualiser "comme si» il s'agissait d'une situation de communication réelle, ce qui complexifie le processus de contextualisation. Bien que l'élève doive tenir compte du contexte d'apprentissage (les attentes de l'enseignant, ses consignes et exigences, les nouvelles connaissances apprises, etc.), c'est bien aux paramètres des situations d'écriture qu'ils doivent adapter leur texte. On sera alors amené à se demander comment les élèves contextualisent les situations d'écriture de justification qui leur sont soumises en classe et comment cette contextualisation influence leurs manières d'élaborer leur justification à l'écrit.

\section{Explorer l'activité scripturale par sa verbalisation: une méthodologie centrée sur l'élève et son point de vue}

Delamotte et Penloup (2000) ont montré l'intérêt d'une didactique centrée sur l'apprenant et ses pratiques et la pertinence de recherches sollicitant non pas l'explication, mais plutôt "l'explicitation de manières de faire composant une culture, de modèles d'action caractérisant des usages" (p. 46). Leurs propositions trouvent un écho dans une méthodologie de recherche qualitative et compréhensive qui repose sur deux postulats: celui de la capacité d'un chercheur à saisir, du point de vue des acteurs eux-mêmes, le sens qu'ils donnent aux phénomènes étudiés (Paillé et Mucchielli, 2008) et celui de la capacité d'un acteur «à évoquer ce qu'il fait, pense, ressent [...] ainsi qu'à analyser ce que représente pour lui l'acte [évoqué]» (Delamotte et Penloup, 2000, p. 41). Cette posture épistémologique de recherche a mené à placer les élèves au cœur de la stratégie méthodologique de cette 
recherche en sollicitant leur témoignage à propos de ce qui se passe cognitivement et se joue affectivement et socioculturellement chez eux au cours de l'écriture d'une justification en classe de français.

\subsection{Les participants}

La collecte de données s'est déroulée en avril 2012 dans une école de Montréal (Québec, Canada) qui reçoit des indices élevés de défavorisation. Plus de $60 \%$ des élèves qui fréquentent cette école francophone sont en situation de langue seconde et $40 \%$ d'entre eux sont issus de l'immigration. Quinze élèves provenant de trois classes ont participé à cette recherche: cinq de première secondaire et dix de deuxième secondaire. Ce groupe comptait huit garçons et sept filles, huit allophones et sept francophones, huit élèves nés au Québec et sept à l'étranger. Tous ces élèves fréquentent par ailleurs la classe ordinaire de français depuis au moins deux ans, ne sont pas en situation de difficulté scolaire, recourent spontanément et avec aisance au français dans les interactions orales et sont plurilingues puisqu'ils «maitrisent, à des degrés divers, des langues et ont, à des degrés divers, l'expérience de diverses cultures" (Coste, Moore et Zarate, 1997, p. v).

\subsection{La production des données}

La méthode de l'entretien d'explicitation (EdE) (Vermersch, 1996) a été choisie parmi toute une panoplie de types d'entretiens visant la verbalisation de l'action ${ }^{4}$. Cette méthode vise une description aussi fine et soignée que possible d'actes passés, en s'appuyant sur le seul support mnésique, ce qui constitue un avantage pour qui souhaite maintenir les participants dans les conditions les plus naturelles et habituelles possibles, ce que ne permettent pas d'autres méthodes de verbalisation qui requièrent divers appareillages de captation ou d'enregistrement. Le but principal de ce type d'entretien pour la recherche consiste à s'informer de ce qui s'est réellement passé du point de vue de l'acteur et de faire émerger les connaissances implicites inscrites dans son action. Pour mener un entretien d'explicitation $^{5}$, la première étape consiste à expliquer au participant le but de la recherche,

Une recension de méthodes et techniques de verbalisation de l'action et un examen approfondi de leurs limites et de leur potentiel ont été réalisés (voir à cet effet Forget, 2013).

5 Cette méthode d'entretien, très technique, demande une formation et beaucoup d'entrainement, ce à quoi la chercheuse s'est employée. L'EdE, s'il est bien mené, produit des données très fines et plus précises que la technique des protocoles verbaux par exemple (Gufoni, 1996). 
puis à lui proposer un contrat qui cadre l'intention de l'entretien: «Nabyl, es-tu d'accord pour me parler de comment tu fais quand tu...". Une fois le contrat établi, on propose à l'acteur de choisir une activité en lien avec la recherche et qu'il a effectivement réalisée. L'intervieweur guidera ensuite l'évocation de la situation par un questionnement assez dirigé (Qui d'autre est là? Où es-tu placé? Comment te sens-tu? Que vois-tu? Qu'entends-tu? Que se passe-t-il à ce moment? À quoi penses-tu? etc.). Ce questionnement aide le participant à se replacer dans un "quasi-revivre" de la situation qu'il évoque. Quand la personne "y est", son discours passe au "je», elle se centre sur ce qu'elle a fait et décrit son activité à l'aide de verbes au présent, indices qu'elle est placée en "position de parole incarnée». Le chercheur peut alors orienter l'évocation de l'activité sur la description fine des actions effectuées (Et là, que fais-tu? Et ensuite? Et après?) plutôt que sur leur explication ou leur justification qui donnent, elles, davantage accès à des représentations. Puis, des moments de son activité sont ciblés parce qu'ils apparaissaient comme étant cruciaux pour répondre aux questions de la recherche. Le participant est alors amené à focaliser son attention sur ces moments, à les fragmenter et à expliciter les actions, très fines, qu'il avait réalisées, un peu comme s'il ouvrait des poupées russes. Précisons que le questionnement se réalise "à partir» et "avec" les mots de l'acteur: il s'agit de relancer le participant sur l'explicitation de son action. En voici un exemple:

Chercheur: Et ensuite tu fais quoi?

Participant: Je l'écris sur ma feuille

C: Et quand tu l'écris sur ta feuille qu'est-ce que tu fais?

P: J'essaie de formuler la fin de la phrase pour qu'elle aille bien avec le commencement

C: Pour qu'elle aille bien avec le commencement. Comment tu sais que ça va bien avec le commencement? P: ...

Les EdE ont produit des données qui ont, entre autres, permis de décrire avec précision comment des élèves ont contextualisé les situations d'écriture qui leur ont été soumises et comment ils ont procédé à l'élaboration du contenu de leurs textes justificatifs.

Pour situer les données issues des entretiens dans leur contexte et mettre à l'épreuve le travail d'analyse des verbatim (triangulation), des notes de terrain ont été produites à partir 
de l'observation directe (Laperrière, 2008), dans chacune des classes, d'environ sept séances de 75 minutes au cours desquelles a eu lieu l'enseignement de l'écriture de justification. Au terme de ces séances, une situation d'écriture de justification était soumise aux élèves. Ce sont ces situations d'écriture qui ont fait l'objet d'une explicitation dans le cadre des entretiens. Tout le matériel didactique utilisé ainsi que les productions (exercices, notes, plans, brouillons, textes finaux) des 15 élèves participants ont été recueillis.

\subsection{L'analyse des données}

Les données d'entretien ont été analysées selon la méthode d'analyse par théorisation (Paillé et Mucchielli, 2008) qui consiste à catégoriser les phénomènes émergeant des données brutes et à les mettre en relation afin d'en découvrir et d'en comprendre les interactions. La mise en place de la théorisation dans le cadre de cette étude a adopté une stratégie empirique $^{6}, c^{\prime}$ est-à-dire que la légitimité des interprétations de la chercheuse devait s'appuyer sur leur fidélité aux données brutes, et a suivi grosso modo les étapes qui seront maintenant décrites. Les entretiens ont d'abord été retranscrits à la suite de quoi ils ont été codifiés. La codification consiste à nommer, à l'aide de mots-clés, les phénomènes qui apparaissent en lien avec le cadrage et les objectifs de la recherche au cours de nombreuses lectures comparatives. Les extraits codifiés de manière semblable sont ensuite importés dans une fiche analytique afin de procéder à la catégorisation des phénomènes. La catégorisation consiste, à partir de l'analyse inductive des extraits, à définir le phénomène, à en identifier les propriétés ainsi que les conditions d'existence c'est-à-dire les conditions sans lesquelles le phénomène n'apparait pas. Voici l'exemple d'une fiche analytique présentant une tactique explicitée par l'ensemble des élèves interrogés:

6 Deux autres stratégies sont possibles: la stratégie théorique (mise à l'épreuve à partir des savoirs légitimes du domaine) et la stratégie spéculative (vérification d'intuitions, de preconceptions, voire d'hypothèses). 


\section{Tableau 1}

Exemple de fiche analytique des catégories conceptualisantes

\begin{tabular}{|c|c|}
\hline Catégorie & Autoquestionnement \\
\hline Définition & $\begin{array}{l}\text { Action de se poser la question qui sous-tend la position à justifier (pourquoi dis-je } \\
\text { que...?) de manière à générer les raisons à l'appui en répondant pour soi-même à } \\
\text { la question. }\end{array}$ \\
\hline Propriétés & $\begin{array}{l}\text { - Soliloque intérieur sous forme de question/réponses } \\
\text { - Questionnement portant sur la position à justifier } \\
\text { - Récurrence de la question autant que nécessaire à l'établissement des raisons } \\
\text { - Questionnement qui structure le raisonnement justificatif (parfois sa mise en } \\
\text { texte) } \\
\text { - Questionnement qui génère les éléments à l'appui de la position }\end{array}$ \\
\hline $\begin{array}{l}\text { Conditions } \\
\text { d'existence }\end{array}$ & $\begin{array}{l}\text { - Toute tâche d'écriture de justification } \\
\text { - Position établie d'emblée sur la question à justifier } \\
\text { - Raisons à l'appui indéterminées }\end{array}$ \\
\hline Extraits & $\begin{array}{l}\text { Mar: C'est comme dans ma tête je me dis «pourquoi il est important de se } \\
\text { rappeler de cette maladie» pis j'essaie de me rappeler ce que j'ai lu dans le texte } \\
\text {--- } \\
\text { Da: Et là, «l'héritage culturel c'est important, pourquoi c'est important?», donc } \\
\text { le mot pourquoi il revient souvent dans ma tête }\end{array}$ \\
\hline
\end{tabular}

Une fois les catégories conceptualisées, on procède à leur mise en relation, c'est-à-dire qu'on va chercher et nommer les liens qui les relient. Suit l'étape d'intégration des ensembles de catégories mises en relation. De cette intégration découle une proposition de sens qui, ancrée dans les données d'entretiens, vise à rendre compte du fonctionnement du phénomène étudié du point de vue des acteurs.

\section{La contextualisation et les actions qui en découlent: éléments de théorisation}

Les situations d'écriture de justifications que les 15 élèves interrogés ont choisi d'évoquer lors des entretiens peuvent être regroupées selon les trois types de justification que l'on trouve habituellement en classe de français (voir section 2). Le premier concerne l'écriture de réponses à des questions liées à la lecture de textes littéraires. Il s'agit alors davantage de tâches de lecture qui sollicitent l'écriture de réponses demandant à être justifiées. Le second type regroupe deux tâches d'écriture de textes justificatifs présentant une position 
sur une question: l'écriture d'une critique littéraire et celle d'un dépliant présentant son point de vue sur une question sociale. Le troisième type correspond à l'écriture d'un texte de recommandation: une lettre adressée au Comité international olympique (CIO) dans laquelle les élèves devaient présenter un sport et justifier l'intérêt de le voir ajouté au programme olympique. Pour chacun des trois types de justification et à l'aide d'extraits d'entretiens, seront maintenant illustrées différentes manières avec lesquelles les élèves ont contextualisé la situation d'écriture et ont élaboré leurs justifications.

\subsection{Répondre par écrit à des questions liées à la lecture littéraire}

L'élaboration de la justification d'une réponse à une question liée à la lecture d'un texte littéraire concerne la position de l'élève sur différents aspects du texte (Que penses-tu de la décision du personnage $x$ ? Si tu avais été à la place du personnage aurais-tu agi de la même façon? As-tu aimé ce roman? Pourquoi?). Bien qu'il ne s'agisse pas de situations d'écriture à proprement parler, il apparait intéressant de constater que certains élèves vont contextualiser la situation de référence, c'est-à-dire la situation à propos de laquelle l'élève doit élaborer sa justification écrite, avec beaucoup de précision. En effet, pour parvenir à prendre position sur la question et à générer les raisons qui la fondent, des élèves ont mentionné s'imaginer être à la place du personnage du texte lu. Ariana7 I'explicite de manière éloquente:

M: Et quand tu réfléchis [à la question posée], tu réfléchis comment?

A: C'était des images (M: des images?) oui

M: Elles sont comment les images?

A: Elles sont plutôt obscures (M: obscures) comme si ça se passait dans un endroit fermé...

M: Et donc tu vois des images plutôt obscures et ensuite?

A: Je m'imagine un peu (M: tu t'imagines) oui et... (M: et quand tu t'imagines, tu t'imagines comment?) ben dans la même situation que lui vivait (Ariana).

Ariana révèle ici qu'elle cerne très clairement les contenus référentiels de la situation de référence, c'est-à-dire qu'elle se représente de manière détaillée l'extrait du roman concerné par la question à laquelle elle doit répondre (Si tu avais été à la place du personnage, comment aurais-tu agi?). Et elle va plus loin en indiquant juste après qu'il ne s'agit pas pour 7 Des pseudonymes ont été utilisés pour préserver l'anonymat des élèves. 
elle de s'imaginer la scène de l'extérieur, mais bien de se projeter littéralement à la place du personnage afin de mieux comprendre ce qui lui arrive:

M: Donc tu t'imagines, dans la même situation que lui. Qu'est-ce que tu imagines?

A: Mais, je m'imagine que, j'essaie de dormir et, à la fin je m'échappe (M: et c'est comment ça?) c'est dans le noir

$\mathrm{M}$ : donc c'est dans le noir, et après?

A: ... je pense que je pleure

M: tu te vois pleurer, tu te sens pleurer?

A: oui je me sens plutôt pleurer

M: Comment tu sais que tu pleures?

A: Je sens des larmes

M: OK, et ensuite qu'est-ce que tu fais?

A: Euh... après quelques minutes je, je m'échappe (M: tu t'échappes) oui (Ariana).

Cette projection dans "l'expérience» du personnage va provoquer chez elle des réactions (elle s'imagine qu'elle pleure) qui lui permettent d'établir sa position sur la question de départ et lui fournissent les raisons pour lesquelles elle aurait agi comme le personnage:

A: Mais, je sais que, quand j'ai imaginé (M: oui) je savais que je ne me serais pas sentie bien (M: OK) et c'est pour ça que je l'ai écrit

M: Donc quand tu écris pourquoi tu aurais fait la même chose, tu sais ce que tu vas répondre

A: Oui, j'ai pas besoin d'y réfléchir trop longtemps parce que, je l'avais déjà (Ariana).

Cette tactique d'Ariana montre une capacité à contextualiser, comme si elle y était, la situation de référence, à se représenter le contenu référentiel et à se projeter à la place de l'autre de façon remarquable.

A contrario, Jessy semble se soucier de conventions d'ordre scolaire liées à ce qu'elle pense être, ce qui est attendu d'elle: "elle [l'enseignante] nous donne une question, et là on répond et après ça on justifie là, pourquoi, mettons elle [...] cinq lignes, on remplit les cinq lignes pour le faire» (Jessy). Jessy a du mal à saisir les contenus référentiels concernés par les questions auxquelles elle doit répondre: elle ne se rappelle pas sur quoi portait la 
question dont elle parle ni quelle était sa position. La situation de référence lui échappe. Un peu plus loin elle ajoute: "si tu justifies pas chaque point tu peux perdre des points, justifie chacun de tes points, aies pas peur d'en mettre parce que plus t'en mets, plus t'as de chance d'avoir des meilleurs points là" (Jessy). Ce commentaire montre que Jessy se centre plutôt sur des représentations liées aux exigences scolaires, éléments de la culture éducative qui constituent des paramètres de la situation immédiate de la classe de français. Évoquant des questions contenues dans les manuels scolaires, Janick révèle également une contextualisation limitée à la situation immédiate de la classe:

Dans les exercices de ce cahier-là qui sont interminables, ça là (ellemontre le manuel d'activités), ça y nous demande beaucoup de justification, j'ai pas vraiment d'exemples parce qu'on en a fait beaucoup [...] oui et en plus on en fait souvent de ça, t'sais pendant les cours c'est pas mal ça qu'on fait sauf que Mme $L$ nous a mis un film pour nous récompenser d'avoir fait beaucoup de travail (Janick).

Elle ne s'est rappelée ni une question en particulier, ni sa position sur cette question, ni le texte qui y était associé. Une telle contextualisation semble associée à des préoccupations chez les élèves, telles que remplir le nombre de lignes proposées ou accumuler beaucoup d'éléments à l'appui de sa position sans réelle prise en compte de la situation de référence.

Ce qui vient d'être décrit laisse apparaitre une influence de la contextualisation qu'effectue l'élève de la situation sur sa façon d'élaborer sa justification. Lorsque l'élève focalise sur la situation de référence, ce qui le préoccupe et ce qu'il fait semblent pouvoir favoriser l'élaboration d'une justification mieux fondée.

\subsection{Rédiger des textes pour donner son avis}

Les situations d'écriture explicitées par les élèves relativement à la rédaction de textes visant à justifier une position personnelle sont celles de la critique littéraire et du dépliant promotionnel. Ces élèves ont tous décrit une contextualisation de niveau micro de la situation d'écriture: l'intention de communication ainsi que les contenus référentiels apparaissent clairement. Aucun cependant n'a dit s'être préoccupé des caractéristiques de son destinataire. Or, la dimension dialogique inhérente au discours justificatif demande une capacité à tenir compte du point de vue et des caractéristiques de son interlocuteur 
(Garcia-Debanc, 1996; Golder, 1996). En effet, justifier demande à faire valoir la légitimité de son propos, aux yeux de son interlocuteur.

Pour élaborer sa justification, Carlos s'est remémoré des évènements de sa propre expérience, ce qui lui a servi à prendre position:

Je pense, parce que la question était "qu'est-ce qu'on doit transformer, changer ou garder [et pourquoi]", et je me... demande... quand je suis arrivé ici est-ce que c'était bon, est-ce que j'aimais ça, est-ce que c'était bon pour un immigrant une personne qui a jamais vu ça et j'ai vu que c'était vrai, so j'ai écrit "il faut garder l'école comme ça et rien transformer" parce que au Québec, y a beaucoup de cultures c'est multiculturel, et je pense qu'il ne faut rien changer j'ai écrit ça... j'ai écrit j'ai vu mon expérience (Carlos).

Carlos va également procéder à l'accumulation d'exemples qui confirment sa position, exemples qui proviennent d'évènements vécus par des personnes qu'il connait:

J'ai aussi pensé que j'ai un ami qui vient d'arriver il y a un an et il a déjà un travail ici, il a 15 ans et il travaille déjà, il sait parler français, pas parler comme bien, bien, bien comme un Québécois, mais il sait se défendre.

[...]

j'ai pensé à d'autres personnes qui ont vécu la même chose que moi. [...]

J'ai pensé à mon amie parce que c'était la plus récente arrivée et parfois je la vois triste et parfois joyeuse parce qu'ici on peut sortir, c'est très sécuritaire, c'est pas comme au Venezuela et là j'ai pensé à ça (Carlos).

Ces exemples lui servent alors à formuler les raisons qui appuieront sa position, mais il semble que Carlos ne se préoccupe pas de la recevabilité de sa position ni aux yeux de l'enseignant, ni aux yeux du lectorat correspondant à la situation d'écriture. On peut penser que Carlos a adopté une stratégie cognitive et langagière correspondant à une posture dite "première" révélée par "la mise en mots directe, sans distance, de l'expérience de soi, sorte de transcription au plus près d'un vécu-perçu-imaginé-pensé, trace brute d'une pensée qui émerge» (Bucheton, 2006, p. 34).

À la question: "À qui tu adressais cette critique-là?», Zaïd répond: "à tout le monde qui va la lire, je donnais mon opinion et je racontais pas ça à personne, parce que si, mettons, on le met sur Internet n'importe qui pourrait le voir et il pourrait prendre ça pour lui, donc 
je l'ai écrit pour lui, pour les lecteurs qui vont le lire» (Zaïd). On soupçonne que Zaïd ne s'est pas réellement posé la question de savoir qui était le lecteur potentiel de son texte et comment il devait en tenir compte dans l'élaboration de sa justification. En revanche, il s'intéresse aux exigences de son enseignante: "on a une démarche à suivre dans notre cahier, elle [l'enseignante] nous a expliqué les étapes qu'on devrait utiliser pour bien faire notre critique (M: oui) et il faut qu'on la suive pour que notre critique soit réussie» (Zaïd). Zaïd considère vraisemblablement son enseignante comme étant la véritable destinataire de son texte. S'il a cerné le contenu référentiel de la situation d'écriture, c'est en s'appuyant sur son expérience personnelle et en la comparant avec l'univers du roman qu'il a pu trouver les raisons lui permettant de fonder son avis selon laquelle l'une des qualités du roman est son caractère vraisemblable, son réalisme:

M: Comment tu sais que [l'univers du roman] c'était réel?

Z: Parce que je faisais des, des... un parallèle avec mon passé, et ce que j'ai vécu là-bas ${ }^{8}$ et j'ai fait des comparaisons et j'ai trouvé que ça se ressemblait

M: OK, et quand tu fais une comparaison avec ce que t'avais vécu là-bas comment ça se passe?

Z: Dans ma tête je me souviens de tout ce que j'ai, j'ai vécu là-bas (M: oui)... et après, j'ai fait, des liens avec ce que je lisais en ce moment et là, j'ai fait des comparaisons avec ce que je me rappelais et ce que je lis et ça, ça se comparait

M: On pouvait donc comparer le livre et tes souvenirs, ça se comparait et (Z: oui) et donc t'as choisi, cette raison-là, que c'était comme tu m'as dit

Z: Ça avait l'air réel (Zaïd).

II ressort de cette section que dans des situations d'écriture de justification destinées à faire valoir une position (avis, opinion) sur un objet (roman) ou sur un sujet non controversé, la prise en compte des caractéristiques du destinataire, essentielle à la gestion de la dimension dialogique de ce discours et à l'adaptation du texte aux paramètres de la situation d'écriture, apparait lacunaire. En fait, la justification d'une position apparait pour plusieurs élèves comme une occasion d'affirmer leur identité, de dire qui ils sont et ce qu'ils pensent comme le soulignent ces extraits: "Comme une justification, y a pas de bonnes ou de mauvaises réponses vu que c'est sur ton opinion" (Jessy); "C'est ton opinion que tu

8 Cet élève a grandi en Irak jusqu'à l'âge de neuf ans. L'histoire du roman se situe au Liban, un pays du Moyen-Orient. 
vas écrire, et t'as vraiment le gout d'écrire ça parce que c'est ton OPINION ${ }^{9}$, c'est ton AVIS, c'est toi qui va l'écrire c'est TON TEXTE” (Zaïd); “... Le but c'est pas non plus de satisfaire, c'est plutôt d'expliquer ce que toi tu penses" (Ariana). Si la force d'une position réside dans le choix d'éléments fiables aux yeux du destinataire, il semble que les élèves qui adoptent une posture première ne s'en préoccupent pas tellement. La contextualisation que font les élèves de ce type de situations d'écriture gagnerait à être accompagnée pour les aider à mieux tenir compte de la dimension dialogique propre à la justification.

\subsection{Rédiger des textes pour émettre des recommandations}

L'analyse de l'explicitation de la tâche d'écriture de la lettre adressée aux membres du $\mathrm{CIO}$ concernant l'intégration d'un nouveau sport au programme olympique laisse également apparaitre une influence de la contextualisation qu'effectue l'élève sur sa manière d'élaborer sa justification. Gladys a bien nommé le but de ce texte: faire valoir l'ajout du squash au programme olympique. Or, elle n'a jamais évoqué une prise en compte des paramètres macro et micro de la situation d'écriture. La construction d'une représentation claire des contenus de référence, de ses destinataires et de leurs caractéristiques lui semble inconnue. À la question de savoir ce que fait Gladys pour planifier l'écriture de son texte, elle répond: "oui je sors mon crayon, mon efface, mon surligneur et puis mon aiguisoir, puis je range les autres affaires, il faut que j'aiguise pour que ça soit bien quand je vais commencer» (Gladys). Une telle réponse montre qu'elle a contextualisé la situation immédiate dans laquelle elle se trouvait elle-même en tant qu'élève. Tout au long de son explicitation, Gladys évoquera les consignes de l'enseignant et ses conseils comme le montre cet extrait: “j'écris "ensuite» là j'ai vu que Monsieur L nous avait dit qu'il fallait le mettre so je l'ai mis, pis je pense que ça m'a donné beaucoup de points, d'habitude je le fais pas, mais là dans ce texte-là, je l'ai fait et ça c'est bien passé» (Gladys). Par ailleurs, le moyen qu'elle évoque pour s'assurer d'avoir une bonne note consiste à prier: «À la fin, c'est sûr que... souvent, je fais, je fais ça (elle fait un signe de croix) pour que ça arrive! Comme pour que j'aie une bonne note» (Gladys). Quant à Tashin, il va s'imaginer les réactions de son enseignant pour évaluer la pertinence des raisons qu'il utilise pour appuyer sa proposition:

9 Les mots en lettres capitales indiquent que l'élève a prononcé ce mot avec emphase lors de l'entretien. 
T: Je me dis, si... comme plus que pensable, je pense si Monsieur L va l'accepter ou dire "non c'est pas un bon argument"

M: Et comment tu sais que Monsieur L va dire que c'est un bon argument ou pas?

T: Je pense qu'il serait d'accord avec mon idée (Tashin).

Sa remarque montre une capacité à envisager les réactions d'autrui certes, mais elle semble limitée à sa représentation des exigences de l'enseignant, seul critère disponible pour juger de la recevabilité de ses propos. La stratégie cognitive et langagière adoptée par ces élèves correspond à ce que Bucheton (2006) appelle la posture scolaire: «Tous les efforts d'écriture de l'élève sont tendus vers le souci de respecter ces règles et exigences, dont il s'est construit une représentation" (p. 33). Il apparaît au fil de la théorisation que cette posture semble amener ces élèves à contextualiser les paramètres de la situation immédiate plutôt que les paramètres de la situation d'écriture. Posture et contextualisation semblent avoir une incidence mutuelle.

Yvonne, au contraire, contextualise la situation d'écriture comme si elle y était. Parlant de ses destinataires elle dira:

Je les visualise comme un groupe de personnes importantes, c'est comme si... ils sont devant moi et il faut que je communique avec eux et je peux pas parler comme en cours de récréation, il faut que je pense à ce que je vais dire et là je me mets à leur place et je me dis: "si je disais ça, qu'est-ce que j'en penserais si j'étais une d'euX"? Et là je l'ai écrit (Yvonne).

Cette tactique lui permet vraisemblablement de se construire une représentation juste et claire de son destinataire. Elle mentionne d'ailleurs s'être documentée sur le mouvement olympique pour mieux comprendre le fonctionnement et les valeurs de cette institution:

[...] j'avais cherché ce que c'était le mouvement olympique l'importance que ça avait le but des JO c'est quoi aussi, il faut se demander, alors je me suis dit le mouvement olympique c'est bon, le fait de faire du sport dans le respect et tout et de dire que mon sport il représente bien ça, ça c'est vraiment un bon argument (Yvonne).

Elle témoigne également d'une préoccupation importante du statut et du rôle de chacun des interlocuteurs dans l'établissement de sa position énonciative: «[...] alors je me disais 
est-ce que je suis juste une personne qui essaie comme tant d'autres de proposer le squash dans les JO ou bien est-ce que je suis la représentante de la fédération du sport» (Yvonne). La situation immédiate de la classe avec toutes ses contingences institutionnelles ne semble plus exister pour Yvonne qui n'y a fait aucune allusion tout au long de l'explicitation de l'élaboration de sa justification. Seule semble exister pour elle son interaction imaginée avec les membres du CIO. De surcroit, elle a en quelque sorte reconstitué le niveau macro de la situation d'écriture en s'informant plus avant sur la culture de cette institution et le statut de ses membres. Aussi, contrairement à plusieurs de ses pairs, Yvonne semble posséder des critères pour évaluer le contenu de son texte tout autant que sa forme:

C'est pas le même vocabulaire par exemple (M: qu'est-ce que tu veux dire?) si j'essaie de convaincre une pauvre dans la rue de m'acheter cette nourritureousij'essaiedevendreàunegrandeentreprisejevaispasutiliser les mêmes termes, encore là, il faut, les arguments qui vont lui servir à cette personne. Tu sais que ça va pas être les mêmes arguments (Yvonne).

Enfin, Yvonne mentionnera ceci: «J'étais stressée de réussir et de pouvoir trouver les mots à l'examen [...] donc j'ai considéré le projet plus important parce que c'était un examen et ça allait beaucoup compter alors je me suis, on pourrait dire, plus appliquée» (Yvonne). Au-delà de considérations motivationnelles quant à la réussite de cette tâche, ce commentaire mis en parallèle avec l'explicitation de l'écriture de son texte montre que "le JE de l'énonciation ne se confond pas avec le «je» de l'expérience» (Bucheton, 2006, p. 35). Contrairement à Gladys et Tashin pourtant confrontés à la même situation, Yvonne sait faire la différence entre son rôle d'élève et les enjeux qui s'y rattachent dans la situation immédiate de la classe, et son rôle d'énonciatrice dans la situation d'écriture. La démarche d'analyse par théorisation montre un lien entre posture et contextualisation: la capacité à bien contextualiser les niveaux macro et micro d'une situation d'écriture va de pair avec l'adoption d'une posture seconde attestée par la capacité à se distancier de son texte, à en évaluer les aspects discursifs, textuels et linguistiques à partir de connaissances, à questionner ses stratégies ou à tenir compte d'autres points de vue (Bucheton, 2006). Les élèves qui adoptent une posture seconde semblent plus aptes à imaginer la situation de référence, à se projeter à la place de leur destinataire, à se questionner sur la pertinence des éléments d'étayage en appui sur les caractéristiques du destinataire, à bien se représenter leur rôle et leur statut dans l'énonciation et à adopter une voie énonciative cohérente, 
ce qui favorise potentiellement l'élaboration de justifications plus efficaces. En effet, selon l'observation des productions des élèves, quand l'élève adopte une posture seconde, sa justification se montre plus pertinente et son texte est de meilleure qualité ${ }^{10}$.

\subsection{La contextualisation en situation d'écriture: synthèse de la théorisation en état}

Loin de vouloir éluder ou récuser les facteurs liés aux spécificités du mode écrit par rapport au mode oral pour expliquer les difficultés rencontrées par les élèves dans l'écriture de textes justificatifs, il apparait que leurs manières de contextualiser les situations d'écriture de justification qui leur sont soumises influencent, favorablement ou non selon les cas, l'élaboration de leurs justifications. La figure suivante en présente la synthèse:

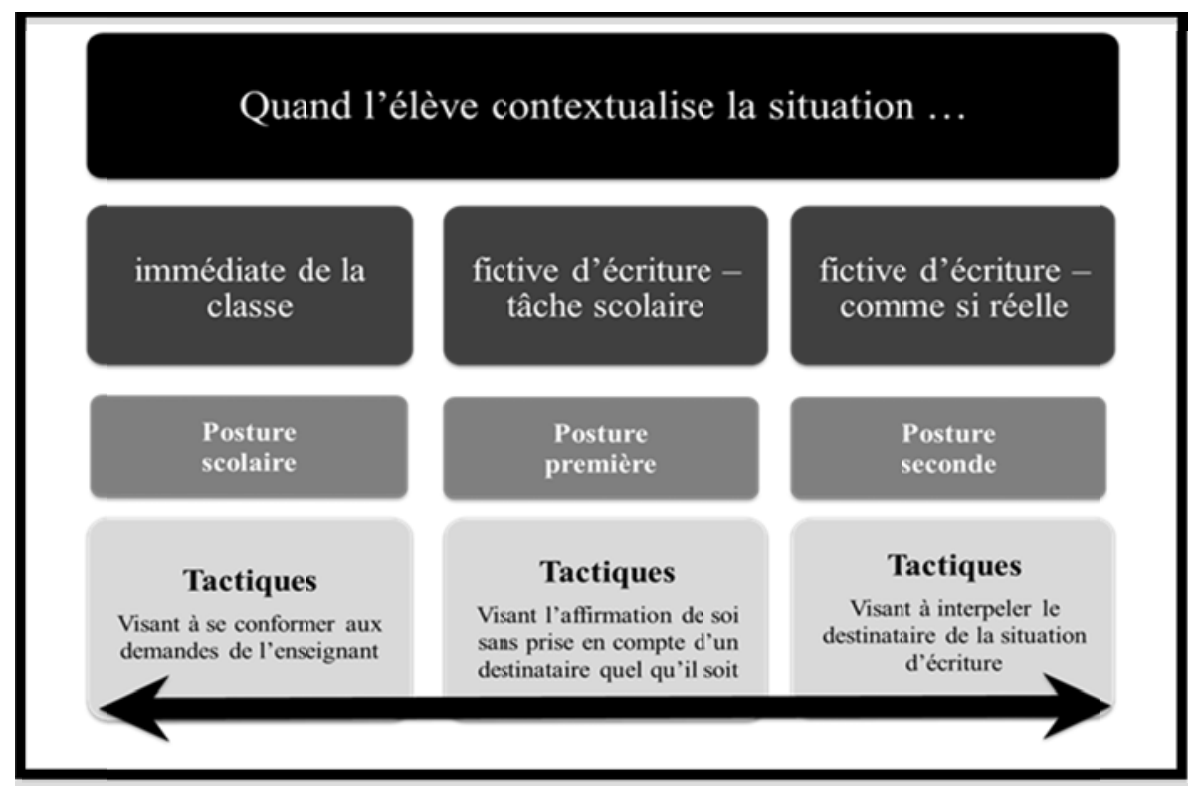

Figure 1. Synthèse des liens entre contextualisation, posture et actions

Quand l'élève ne se préoccupe que de la situation d'apprentissage immédiate (celle dans laquelle il est lui-même placé en tant qu'élève), il adopte une posture dite scolaire qui le mène à se conformer aux exigences institutionnelles qu'il se représente dans le but d'obtenir de bons résultats (accumuler les raisons à l'appui de sa position, se demander si son enseignant serait d'accord avec lui, etc.). Lorsque l'élève se préoccupe de la situation

10 Les productions des élèves (notes, plans, brouillons et textes finaux) n'ont pas fait l'objet d'une analyse systématique. Ainsi, cette observation doit faire l'objet d'une analyse en bonne et due forme avant de pouvoir conclure à l'existence d'un lien clair entre l'adoption d'une posture seconde et la qualité des textes justificatifs, même si, à priori, il semble que ce soit le cas. 
d'écriture (celle qui constitue le prétexte à l'apprentissage), il a plutôt recours à des tactiques lui permettant d'adapter son discours à cette situation. Certains mettront alors en valeur leur position, leur réponse ou leur proposition à partir d'une posture dite première qui les amènera à vouloir affirmer leur position sans se préoccuper de la faire valoir aux yeux de leurs destinataires. Plus rarement, des élèves vont faire fi de la situation d'apprentissage immédiate. Ils contextualisent la situation d'écriture en recourant à une forme de mise en scène qui consiste à se projeter à la place du protagoniste de la situation de référence, comme l'a relaté Ariana, ou à imaginer une situation d'interaction orale avec le destinataire de la situation d'écriture, comme l'a évoqué Yvonne. Cette mise en scène les aide, selon leurs dires, à prendre position, à générer les raisons qui l'appuient et à en valider la pertinence, en fonction du destinataire du texte. Ce phénomène fait écho à l'une des thèses de Vygotski (1997) qui considère que le dialogue, première forme de langage apprise, précède, sur le plan ontogénétique, le monologue, plus complexe en termes de fonction psychique, parce que demandant une capacité de décontextualisation et de monogestion du discours. Le dialogue étant, pour les élèves, un outil déjà là, la mise en scène d'interactions orales lors de l'écriture de justifications pourrait être l'indice d'un recours à des savoirs d'expérience efficaces pour monogérer la dimension dialogique inhérente à la conduite de justification, manifestant ainsi la mise en place de fonctions psychiques supérieures spécifiques à l'écriture (Vygotski, 1997) nécessaires au développement de compétences scripturales de justification.

\section{Conclusion: des retombées didactiques}

Cet article a mis en lumière combien les situations d'écriture de justification soumises aux élèves en classe de français sont difficiles à contextualiser. Elles demandent, de la part des élèves, de prendre en compte des paramètres de situations fictives, prétextes à l'apprentissage, ainsi que l'élaboration de justifications sans le concours du destinataire dont le rôle est pourtant de juger de l'acceptabilité de son propos. L'analyse des entretiens a fait ressortir que les élèves capables de poser un regard analytique et critique (posture seconde) sur l'élaboration de leur justification écrite sont aussi ceux qui contextualisent, avec le plus de réalisme, les niveaux macro et micro des situations d'écriture qui leur sont soumises en les imaginant sous la forme d'interactions orales. Ainsi, pour aider les autres 
élèves à développer une posture de plus en plus seconde et, ce faisant, à faire progresser leurs compétences scripturales de justification, une attention particulière devrait être portée à leur processus de contextualisation des situations d'écriture. Concrètement, il s'agirait d'accompagner les élèves dans l'interprétation des paramètres des situations d'écriture, d'abord en les aidant à mieux distinguer la situation immédiate de la classe et la situation d'écriture à laquelle on souhaite les voir adapter leur texte. Pour ce faire, un exercice de mise en scène des paramètres de la situation d'écriture pourrait amener les élèves à mieux se la représenter. L'anticipation des réactions plausibles de leur destinataire, la mise à l'épreuve de leur position et des raisons qu'ils souhaitent convoquer pour les appuyer ${ }^{11}$ devrait passer par une verbalisation, donnant accès non seulement à la contextualisation, mais également à la démarche cognitive (le raisonnement) effectuée par les élèves dans l'élaboration de leurs justifications, ce qui permettrait à l'enseignant d'offrir la médiation requise (Bruner, 2008).

Si cette proposition didactique a le mérite de s'appuyer sur des pratiques déclarées et effectives d'élèves du $1^{\text {er }}$ cycle du secondaire, elle ne tient pas compte de la qualité des textes produits par les élèves, dont une analyse systématique aurait sans doute permis de pousser plus loin la compréhension des liens entre "posture", "contextualisation" et "compétence". Si cette limite se doit d'être dépassée, ce que des recherches ultérieures permettront de faire, l'état d'avancement de cette théorisation aura tout de même permis de soulever que si la classe est "un lieu essentiel d'appropriation durable, consciente et transférable [des compétences langagières]» (Blanchet, 2009, n.p.), l'apprentissage de la contextualisation, trop peu pris en considération à l'heure actuelle, devient incontournable pour soutenir les élèves dans le développement de leur compétence scripturale de justification.

\section{Références}

Adam, J.-M. (1996). L’argumentation dans le dialogue. Langue française, 112(1), 31-49.

Blanchet, P. (2007). Quels «linguistes" parlent de quoi, à qui, quand, comment et pourquoi? Pour un débat épistémologique sur l'étude des phénomènes linguistiques. Dans P. Blanchet, L.-J. Calvet et D. de Robillard (dir.), Un siècle après Saussure: Ia linguistique en question (p. 229-294). Paris: L'Harmattan.

11 Le lecteur intéressé par des propositions très concrètes pourra consulter la référence suivante: Forget, M.-H. (2014). Apprendre à justifier. Québec français, 171, 103-104. 
Blanchet, P. (2009). "Contextualisation didactique", de quoi parle-t-on? Le français à l'université. Repéré à http://eprints.aidenligne-francais-universite.auf.org/147/1/pdf. pdf.

Brassart, D. (1990). Le développement des capacités discursives chez l'enfant de 8 à 12 ans: le discours argumentatif écrit. Revue française de pédagogie, 90, 31-41.

Bruner, J. (2008). L'éducation, entrée dans la culture: les problèmes de l'école à la lumière de la psychologie culturelle. Paris: Retz.

Bucheton, D. (2006). Les postures d'écriture et de lecture: la diversité des modes de penser - parler - apprendre. Langage et Pratiques, 37, 29-39.

Bulea, E. et Bronckart, J. P. (2005). Coda: pour une approche dynamique des compétences (langagières). In J. P. Bronckart, E. Bulea et M. Pouliot (dir.), Repenser l'enseignement des langues: comment identifier et exploiter les compétences? (p. 193-227). Villeneuve d'Ascq: Presses universitaires du Septentrion.

Bulea, E. et Jeanneret, T. (2007). Compétence de communication, processus compétentiel et ressources: les apports des sciences du travail et des sciences du langage. In M. Verdelhan-Bourgade (dir.), Le français langue seconde: un concept et des pratiques en évolution (p. 85-110). Bruxelles: De Boeck.

Castellotti, V. et Moore, D. (2008). Contextualisation et universalisme. Quelle didactique des langues pour le XXIe siècle? In P. Blanchet, D. Moore et S. Asselah Rahal (dir.), Perspectives pour une didactique des langues contextualisée (p. 183-203). Paris: Éditions des archives contemporaines.

Chartrand, S.-G. (2013). Enseigner à justifier ses propos de l'école à l'université. Correspondance, 19(1). Repéré à http://correspo.ccdmd.qc.ca/Corr19-1/2.html.

Chartrand, S.-G. et Blaser, C. (2008). Du rapport à l'écriture au concept didactique de capacités langagières: apports et limites de la notion de rapport à l'écrit. In S. G. Chartrand et C. Blaser (dir.), Le rapport à l'écrit: un outil pour enseigner de l'école à I'université (Vol. 12, p. 107-127). Namur: Presses universitaires de Namur.

Chevallard, Y. (2003). Approche anthropologique du rapport au savoir et didactique des mathématiques. In S. Maury et M. Caillot (dir.), Rapport au savoir et didactiques (p. 81-104). Paris: Éditions Fabert.

Coste, D., Moore, D. et Zarate, G. (1997). Compétence plurilingue et pluriculturelle. Vers un cadre européen commun de référence pour l'enseignement et l'apprentissage des langues vivantes. Strasbourg: Éditions du Conseil de l'Europe. 
Delamotte, R. et Penloup, M.-C. (2000). Le passage à l'écriture. Dans R. Delamotte, F. Gippet, A. Jorro et M.-C. Penloup (dir.), Passages à l'écriture. Un défi pour les apprenants et les formateurs (p. 11-105). Paris: Presses universitaires de France.

Dolz, J. (1995). L'apprentissage des capacités argumentatives: étude des effets d'un enseignement systématique et intensif du discours argumentatif chez des enfants de 11-12 ans. Bulletin suisse de linguistique appliquée, 61, 137-169.

Fasel-Lauzon, V. (2014). Comprendre et apprendre dans l'interaction. Les séquences d'explication en classe de français langue seconde. Bruxelles: Peter Lang.

Forget, M.-H. (2012). Qu'est-ce que justifier? . Québec français, 167, 61-62.

Forget, M.-H. (2013). Le développement des méthodes de verbalisation de l'action: un apport certain à la recherche qualitative. Recherches qualitatives, 32(1), 57-80.

Forget, M.-H. (2014). Apprendre à justifier. Québec français, 171, 103-104.

Garcia-Debanc, C. (1994). Apprendre à justifier par écrit une réponse: analyses linguistiques et perspectives didatiques. Pratiques, 84, 5-40.

Garcia-Debanc, C. (1996). Apprendre à justifier à l'école et au collège: ruptures ou continuité? In J. David et S. Plane (dir.), L'apprentissage de l'écriture de l'école au collège (p. 105-130). Paris: Presses universitaires de France.

Goetz, P.-J. et Shatz, M. (1999). When and How Peers Give Reasons: Justifications in the Talk of Middle School Children. Journal of child language, 26(3), 721-748.

Golder, C. (1996). Le développement des discours argumentatifs. Neuchâtel: Delachaux et Niestlé.

Gombert, A. (1998). Comment les rédacteurs de 10 à 13 ans se justifient-ils et argumentent-ils? Rôle du thème rédactionnel, de l'opinion consensuelle et de la thèse défendue. (Université de Provence, Aix-en-Provence).

Gombert, A. (2003). Comment faciliter l'argumentation au primaire. In E. Peyronnet (dir.), Je parle, tu parles, nous apprenons: Coopération et argumentation au service des apprentissages (p. 263-274). Bruxelles: De Boeck.

Gufoni, V. (1996). Les protocoles verbaux comme méthode d'étude de la production écrite: Approche critique. Études de linguistique appliquée, 101, 20-32. 
Hornberger, N. et Skilton-Sylvester, E. (2003). Revisiting the continua of biliteracy: International and critical perspectives. Dans N. Hornberger (dir.), Continua of Biliteracy: An Ecological Framework for Educational Policy, Research, and Practice in Multilingual Setting (p. 35-67). Clevedon: Multilingual Matters.

Kuhn, D. et Udell, W. (2003). The development of argument skills. Child development, 74(5), 1245-1260.

Kyratzis, A., Shuqum-Ross, T. et Koymen-Bahar, S. (2010). Validating justifications in preschool girls' and boys' friendship group talk: implications for linguistic and sociocognitive development. Journal of Child Language, 37, 115-144.

Laperrière, A. (2008). L'observation directe. Dans B. Gauthier (dir.), Recherche sociale: de la problématique à la collecte de données (p. 311-336). Québec: Presses de l'Université du Québec.

Masseron, C. (2003). Conseiller un film d'horreur... justifications et jugements de valeur dans quelques copies de secondes. Pratiques, 117-118, 219-240.

Ministère de l'Éducation, du Loisir et du Sport (2004). Programme de formation de l'école québécoise: secondaire 1er cycle. Québec: Gouvernement du Québec.

Ministère de l'Éducation, du Loisir et du Sport (2009). Épreuve obligatoire, Français, fin du $1^{\text {er }}$ cycle secondaire. Québec: Gouvernement du Québec.

Ministère de l'Éducation, du Loisir et du Sport (2011). La progression des apprentissages au secondaire: Français, langue d'enseignement. Québec: Gouvernement du Québec.

Ministère de I'Éducation du Québec (1995). Programme de français: enseignement secondaire. Québec: Gouvernement du Québec.

Moore, D., Sabatier, C., Jacquet, M. et Masinda, M. (2008). Voix africaines à l'école de la francophonie canadienne. Réflexion pour une culture didactique du plurilinguisme contextualisée. In P. Blanchet, D. Moore et S. Asselah Rahal (dir.), Perspectives pour une didactique des langues contextualisée (p. 19-40). Paris: Éditions des archives contemporaines.

Paillé, P. et Mucchielli, A. (2008). L'analyse qualitative en sciences humaines et sociales. Paris: Armand Colin.

Penloup, M.-C., Chabanois, M. et Joannidès, R. (2011). la posture: effet de mode ou concept pour la didactique du français? Dans B. Daunay, Y. Reuter et B. Schneuwly (dir.), Les concepts et les méthodes en didactique du français (p. 151-174). Namur: Presses universitaires de Namur. 
Porquier, R. et Py, B. (2004). Apprentissage d'une langue étrangère: contextes et discours. Paris: Didier.

Reuter, Y. (2001). La "prise en compte" des pratiques extrascolaires de lecture et d'écriture: problèmes et enjeux. Repères, 23, 9-31.

Vermersch, P. (1996). L'entretien d'explicitation. Paris: ESF.

Vygotski, L.-S. (1997). Pensée et langage. Paris: La dispute.

Wasim, N. (2007). Undergraduates and the Development of Argument. Education Review, $4(3), 31-38$. 